\title{
Fratura de mandíbula tratada através da técnica de Champy: relato de caso
}

Mandibular fracture treated by Champy technique: case report

Fractura de la mandíbula tratada con la técnica Champy: reporte de caso

Éwerton Daniel Rocha RODRIGUES ${ }^{1}$

Carla Cecília Lira Pereira de CASTRO²

Thalita Medeiros MELO ${ }^{3}$

Alan Leandro de Carvalho FARIAS ${ }^{4}$

Laurindo de Sousa BRITO JÚNIOR ${ }^{5}$

${ }^{1}$ Departamento de Cirurgia e Traumatologia Bucomaxilofacial, Hospital Universitário Oswaldo Cruz - HUOC, Universidade de Pernambuco - UPE 50100-130 Recife-PE, Brasil

${ }^{2}$ Faculdade de Odontologia - FOP/UPE, Universidade de Pernambuco - UPE, 50100-130 Recife - PE, Brasil

${ }^{3}$ Faculdade de Medicina FAMED, Universidade Federal de Alagoas - UFAL, 57072-900 Maceio - AL, Brasil

${ }^{4}$ Universidade Federal do Piauí - UFPI, 64202-020 Parnaíba - Pl, Brasil

${ }^{5}$ Hospital Getúlio Vargas - HGV, 64001-020 Teresina - PI, Brasil

\section{Resumo}

Os traumas faciais representam um dos mais importantes problemas de saúde pública. As fraturas mandibulares compõem grande parte das injúrias sofridas pelo complexo maxilomandibular neste panorama. A região mais acometida é o ângulo mandibular (AM), devido a sua área de seccção transversal e biomecânica, além da presença de terceiros molares. Este artigo visa relatar um caso clínico de fratura mandibular, evidenciando diagnóstico e terapêutica. O relato de caso trata-se de um paciente do gênero masculino, 19 anos de idade, que compareceu ao ambulatório do serviço de Cirurgia Bucomaxilofacial de um hospital público queixando-se de dor à mastigação e dificuldade de abertura bucal. Ao exame radiográfico foi identificada solução de continuidade na região de ângulo mandibular, confirmando a hipótese diagnóstica de fratura de mandíbula. O tratamento proposto foi de procedimento cirúrgico sob anestesia geral para osteossíntese através do método retratado por Champy, utilizando-se de acesso intrabucal na região da linha oblíqua externa e bloqueio maxilo-mandibular (BMM) transoperatório com parafusos, seguida a fixação com a utilização de uma placa do sistema 2.0mm e posterior remoção do BMM. O paciente foi acompanhado durante seis meses e mostrou resultado clínico satisfatório. Conclui-se que a técnica de Champy é um método eficaz de fixação interna de fraturas, pois reduz o tempo cirúrgico e a dissecção tecidual, diminuindo as chances de lesões nervosas e complicações pós-operatórias.

Descritores: Mandíbula; Traumatismos Mandibulares; Fixação Interna de Fraturas.

\section{Abstract}

Facial traumas represent one of the most important public health problems. Mandibular fractures make up a large part of the injuries suffered by the maxillomandibular complex in this panorama. The most affected region is the mandibular angle (AM), due to its cross-sectional and biomechanical area, in addition to the presence of third molars. This article aims to report a clinical case of mandibular fracture, showing diagnosis and therapy. The case report is about a 19-year-old male patient, who attended the outpatient clinic of the Maxillofacial Surgery service of a public hospital complaining of pain while chewing and difficulty opening his mouth. In the radiographic examination, a continuity solution was identified in the mandibular angle region, confirming the diagnostic hypothesis of mandible fracture. The proposed treatment was a surgical procedure under general anesthesia for osteosynthesis through the method depicted by Champy, using intra-oral access in the region of the external oblique line and Intermaxillary fixation (IMF) transoperative with screws, followed by fixation with the use a $2.0 \mathrm{~mm}$ system board and subsequent removal of the IMF. The patient was followed for three months and showed a satisfactory clinical result. It is concluded that the Champy technique is an effective method of internal fracture fixation, as it reduces surgical time and tissue dissection, decreasing the chances of nerve injuries and postoperative complications.

Descriptors: Mandible; Mandibular Injuries; Internal Fracture Fixation.

\section{Resumen}

Los traumas faciales representan uno de los problemas de salud pública más importantes. Las fracturas mandibulares constituyen una gran parte de las lesiones sufridas por el complejo maxilomandibular en este panorama. La región más afectada es el ángulo mandibular (AM), debido a su área transversal y biomecánica, además de la presencia de terceros molares. Este artículo tiene como objetivo informar un caso clínico de fractura mandibular, mostrando diagnóstico y terapia. El informe del caso es sobre un paciente de sexo masculino de 19 años que asistió a la clínica ambulatoria del servicio de Cirugía Maxilofacial de un hospital público quejándose de dolor al masticar y dificultad para abrir la boca. El examen radiográfico identificó una solución de continuidad en la región del ángulo mandibular, confirmando la hipótesis diagnóstica de fractura mandibular. El tratamiento propuesto fue un procedimiento quirúrgico bajo anestesia general para la osteosíntesis utilizando el método representado por Champy, utilizando el acceso intrabucal en la región de la línea oblicua externa y el bloqueo maxilomandibular (BMM) transoperatorio con tornillos, seguido de la fijación con el use del una placa del sistema de $2.0 \mathrm{~mm}$ y la posterior eliminación del BMM. El paciente fue seguido durante seis meses y mostró un resultado clínico satisfactorio. Se concluye que la técnica Champy es un método efectivo de fijación de fracturas internas, ya que reduce el tiempo quirúrgico y la disección de tejidos, disminuyendo las posibilidades de lesiones nerviosas y complicaciones postoperatorias.

Descriptores: Mandíbula; Lesiones Mandibulares; Fijación de Fractura Interna.

\section{INTRODUÇÃo}

Injúrias ao complexo maxilo-mandibular representam um dos mais importantes problemas de saúde no mundo. Um interesse particular é criado pela alta incidência e diversidade de lesões faciais ${ }^{1}$. Dentre as fraturas faciais, a mandíbula é um dos ossos mais afetados ${ }^{2,3}$, sendo o ângulo mandibular (AM) a área mais acometida em muitos estudos $^{2,4,6}$, com uma prevalência variando de
23 a $42 \%{ }^{5}$. Para Ellis ${ }^{7}$ a alta taxa de fraturas de AM pode ser explicadas por 3 razões: presença de terceiros molares, área de secção transversal menor que a região dentada e pelo fato de, biomecanicamente, o ângulo ser considerado uma área de "alavanca". Além disso, nessa região há uma mudança abrupta na forma mandibular, de horizontal para vertical, sujeitando o ângulo a forças mais complexas ${ }^{7}$. 
A biomecânica e a posição posterior fazem o tratamento das fraturas nessa região desafiador e, não surpreendentemente, apresentando as maiores taxas de complicações ${ }^{8}$. O tratamento das fraturas de AM pode ser realizado pelo método de redução fechada (com 0 uso de bloqueio maxilomandibular - BMM) ou aberta (com o uso fixação interna - FI) ${ }^{9}$, porém a possibilidade de rotação superior do segmento proximal e as desvantagens da utilização do BMM fazem a redução aberta e a utilização de FI rotineiramente necessárias ${ }^{8}$.

Uma variedade de técnicas tem sido utilizadas para a fixação interna das fraturas de AM, incluindo osteossíntese com fio de aço, 2 placas (borda superior e inferior), uma placa de reconstrução na borda inferior e uma miniplaca de $2.0 \mathrm{~mm}$ na borda superior. A escolha da modalidade de tratamento depende, entre outros fatores, do padrão da fratura ${ }^{10}$. Desde descrição inicial realizada por Michelet et al. ${ }^{11} \mathrm{e}$ do estudo Champy et al. ${ }^{12}$ a utilização de uma miniplaca na borda superior tem sido considerada aceitável ${ }^{13} \mathrm{com}$ uma diminuição da taxa de complicação ${ }^{14}$. Se a utilização de uma miniplaca proporciona a possibilidade de reparação óssea, com complicações pósoperatórias mínimas e simplicidade técnica, certamente haverá economia de custos e de tempo cirúrgico.

Considerando-se a alta prevalência de fraturas de AM, a variedade das formas de tratamento e a alta taxa de complicações póscirúrgicas, o objetivo deste artigo é relatar um caso de tratamento de fratura de AM através do método proposto por Champy.

\section{CASO CLÍNICO}

Paciente do gênero masculino, 19 anos de idade, compareceu ao ambulatório do serviço de Cirurgia Bucomaxilofacial de um hospital público relatando dificuldade de abertura bucal e dor à mastigação. Durante anamnese o mesmo revelou queda da própria altura há 14 dias e não possuir alergias ou doenças de base. Ao exame físico extrabucal não se observava alterações significativas do contorno facial (Figura 1A), havia a presença de lesão abrasiva em região mentoniana em estágio avançado de cicatrização (Figura 1B) e o paciente relatou dor durante à palpação da região goníaca esquerda. Ao exame intra-bucal o paciente apresentava má-oclusão classe I de Angle (Figura 1C), dificuldade de abertura bucal, discreta mobilidade na região de ângulo mandibular esquerdo e dor à manipulação nesta região.

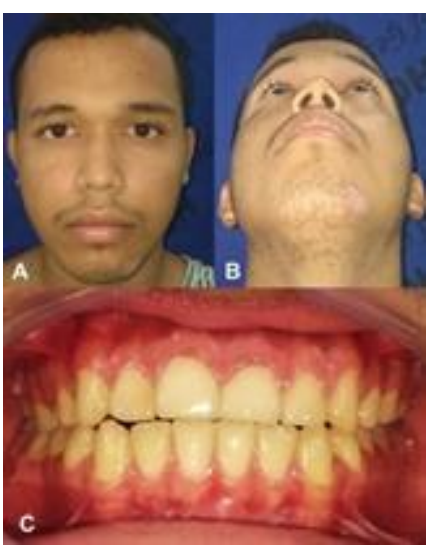

Figura 1: A) Imagem clínica da norma frontal pré-operatória. Imagem clínica da norma frontal caudocranial pré-operatória. C) Imagem clínica da oclusão pré-operatória.

Ao exame radiográfico foi possível observar solução de continuidade na região de ângulo mandibular, confirmando a hipótese diagnóstica de fratura de mandíbula (Figura $2 \mathrm{~A}$ e B).

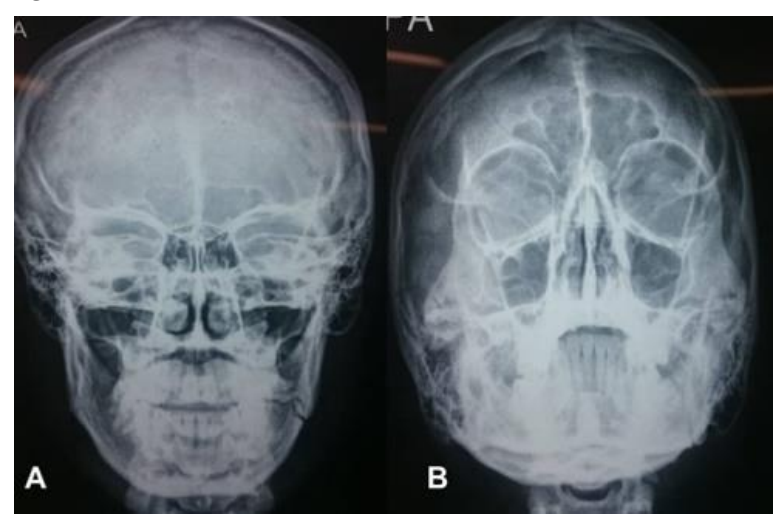

Figura 2: A) Radiografia póstero-anterior de face evidenciando fratura de ângulo mandibular esquerdo. B) Radiografia occiptomentual de face evidenciando fratura de ângulo mandibular esquerdo.

O método de tratamento proposto foi procedimento cirúrgico sob anestesia geral para osteossíntese, através do método proposto por Champy. A cirurgia foi realizada com intubação nasal, acesso intrabucal na região da linha oblíqua externa com descolamento mucoperiosteal total, exibindo a linha de fratura (Figura 3 A). Após a realização do acesso foi realizado 0 BMM transoperatório com parafusos, seguido de fixação por meio de placa do sistema $2.0 \mathrm{~mm}$ do tipo locking, aplicada na linha obliqua externa (Figura 3 B e C).

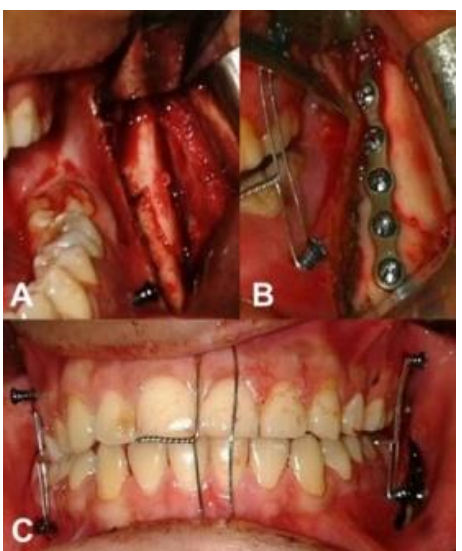

Figura 3: A) Acesso intrabucal exposição da linha de fratura. B) Fixação da fratura com miniplaca dos sistema 2.0 do tipo locking. C) Vista oclusal transoperatória. 
A seguir o BMM foi removido e a oclusão testada, para então, realizar-se a sutura do acesso cirúrgico. O paciente recebeu alta 24 horas após a cirurgia, logo após receber as instruções sobre a higiene e cuidados gerais. $\mathrm{O}$ mesmo retornou após 7 dias para remoção da sutura, e manteve acompanhamento semanal no primeiro mês de pós-operatório. No retorno do sexto mês, observava-se um resultado clínico satisfatório, sem queixas de dor, sem dificuldade de abertura bucal e com cicatrização do acesso cirúrgico satisfatória e radiografia panorâmica evidenciando o reparo da fratura com 0 tempo de pós-operatório (Figura 4 A, B, C. D e E).

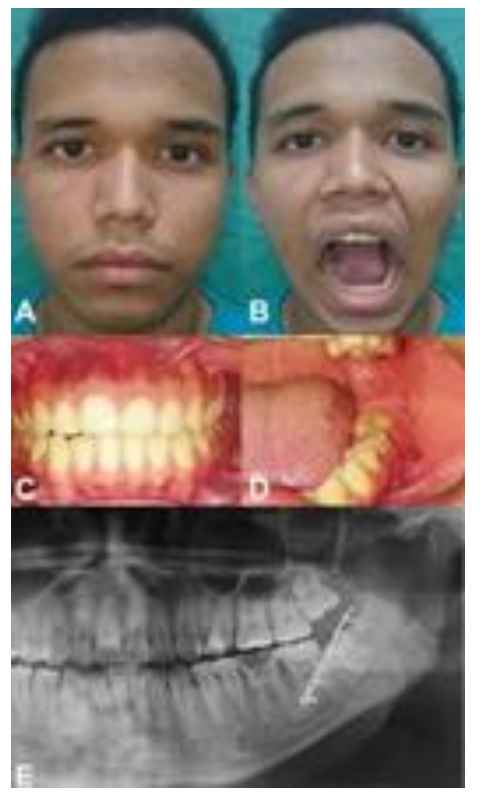

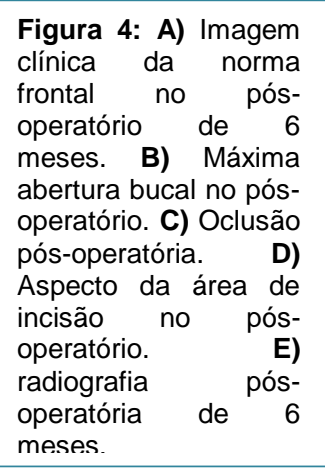

DISCUSSÃO

A literatura indica que as causas mais comuns de fraturas de mandíbula são 0 resultado de conflitos interpessoais ou colisões de veículos motorizados. Nesta discussão, as fraturas mandibulares são categorizadas por mecanismo de lesão e ainda subclassificadas em lesões contundentes de baixa velocidade, penetrantes de baixa velocidade, contundente de alta velocidade e penetrantes de alta velocidade. Isso enfatiza o efeito do mecanismo na apresentação clínica desses tipos de lesões. Quando as fraturas mandibulares são categorizadas por mecanismo, fica claro que existe uma correlação entre o mecanismo, a direção da força e o tipo de fratura ${ }^{3}$.

Fraturas mandibulares são influenciadas por vários fatores, incluindo direção, magnitude da força, padrão de carga oclusal, densidade óssea e até mesmo estruturas anatômicas, como os terceiros molares. Acredita-se que quando um terceiro molar mandibular está presente a zona de tensão é descontinuada, tornando o $\mathrm{AM}$ enfraquecido e, portanto, mais suscetível à fraturas ${ }^{4}$. No estudo retrospectivo publicado por Hasegawa et al. ${ }^{4}$ observou-se que o risco de fratura de AM foi significantemente maior em pacientes com suporte oclusal e com a presença de terceiros molares. Foi possível observar, ainda neste estudo, que não houve correlação significante entre a posição/angulação dos terceiros molares e fratura de AM. Já no estudo realizado por Ma'aita e Alwrikat ${ }^{15}$ relatou-se que o risco de fratura de AM é maior para pacientes com terceiro molares classe 3 posição $C$ e com dentes numa angulação mais vertical, seguida por dentes em posição mesioangular.

Ainda existe um debate acerca dos métodos de fixação para as fraturas de AM, sobretudo pela estabilidade que os esquemas de $\mathrm{FI}$ fornecem. As modalidades de tratamento devem ser estabelecidas considerando as características da fratura. Pacientes que apresentaram fraturas cominutivas, com grande deslocamento e considerável lesão dos tecidos moles devem ser tratados com abordagem extraoral, observando os princípios $\mathrm{AO} / \mathrm{ASIF}^{9}$. Fraturas simples e pouco deslocadas podem ser tratadas pela técnica de Champy et al. ${ }^{12}$. Embora um estudo in vitro tenha demonstrado que a fixação com 2 miniplacas é um método mais estável, com um baixo stress no sítio da fratura, quando comparado com uma única placa instalada na borda superior ${ }^{16}$.

Kim et al. ${ }^{2}$ compararam 3 métodos de tratamento para fraturas de AM (uma miniplaca e BMM, duas miniplacas e BMM e duas miniplacas sem BMM). Neste estudo não houve diferença estatisticamente significante nas taxas de complicações dos métodos aplicados durante os 4 meses de acompanhamento pósoperatório. Por outro lado, Ellis e Walker ${ }^{17}$ mostraram que o uso de duas miniplacas teve uma taxa de complicação de $28 \%$, considerada inaceitável. Em um estudo retrospectivo de 266 fraturas mandibulares, foi relatada uma taxa de infecção $2 \%$ quando se utilizaram placas de $2.7 \mathrm{~mm}$ colocadas extraoralmente. Porém, os pacientes tratados por este método tiveram uma incidência de $8 \%$ de alterações no ramo marginal mandibular do nervo facial no pósoperatório, além de cicatrizes proeminentes ${ }^{18}$.

Um ensaio clínico controlado comparou duas abordagens de fixação de fraturas de AM, no grupo 1 utilizou-se uma placa fixada por acesso intrabucal na linha obliqua externa e no grupo 2, uma placa fixada no aspecto lateral da zona de tensão por meio da combinação de acesso intrabucal e uso de trocarter ${ }^{6}$. Nesse estudo observou-se que houve um índice de deiscência de sutura de $21 \%$ no grupo 1 e de 
9\% no grupo 2 (diferença estatisticamente significante); $36 \%$ dos pacientes do grupo 1 necessitaram de remoção da placa. $\mathrm{Na}$ revisão sistemática publicada por Al-Moraissi e Ellis ${ }^{8}$, quando se comparou a fixação na linha obliqua externa com a fixação no aspecto lateral da zona de tensão foi possível observar uma diferença estatisticamente significante com relação a infecção pós-operatória, deiscência de sutura e falha do material de fixação, com o método de fixação no aspecto lateral da mandíbula apresentando os melhores resultados.

Outra controvérsia envolve o uso do uso do BMM no pós-operatório. Muitos cirurgiões ainda sentem que a fixação com uma única miniplaca não providencia a estabilidade adequada e requer a utilização de $\mathrm{BMM}^{9}$. Em um estudo retrospectivo com 130 pacientes que foram submetidos a fixação de fratura de AM com uma placa fixada de acordo com o método proposto por Champy, dos quais 65 pacientes foram submetidos à utilização pós-operatória de BMM. A taxa de complicação total no grupo que recebeu BMM foi de $17 \%$ e no grupo sem BMM foi de $20 \%$, sem diferença estatisticamente significante ${ }^{19}$.

O manejo dos dentes na linha de fratura também tem sido frequentemente avaliado em relação à incidência de complicações em fratura de AM. A presença de um dente na linha de fratura não requer sua remoção. $O$ dente deve ser removido, no entanto, se dificultar a redução da fratura, se houver processo infeccioso relacionado ao dente, ou houver patologia associada $^{5}$. A desvantagem da remoção rotineira de terceiros molares impactados ou mesmo irrompidos em uma fratura do ângulo mandibular está relacionada à possibilidade de criar um déficit de tecido mole no local de extração. Além disso, existe o risco de converter uma fratura fechada em uma fratura exposta, especialmente quando uma abordagem intrabucal não for utilizada. Outra desvantagem é a necessidade, em potencial, de remover o osso para facilitar sua extração. Isso pode comprometer a qualidade óssea e impossibilitar a fixação de uma placa na linha obliqua externa $^{10}$.

\section{CONSIDERAÇÕES FINAIS}

A fratura do ângulo ainda é uma das fraturas mais comuns da mandíbula e continua associada às maiores taxas de complicações. $O$ tratamento dessas fraturas testemunhou uma mudança significativa nas últimas três décadas. Atualmente, várias técnicas são consideradas aceitáveis para 0 tratamento de fraturas mandibulares. Para fraturas angulares simples e pouco deslocadas, a técnica de Champy é um método eficaz de fixação interna que reduz o tempo cirúrgico e a dissecção tecidual, minimiza o risco para os nervos alveolares inferior e facial e permite o retorno precoce da função com taxas aceitáveis de complicações.

\section{REFERÊNCIAS}

1. Brasileiro BF, Passeri LA. Epidemiological analysis of maxillofacial fractures in Brazil : A 5year prospective study. Oral Surg Oral Med Oral Pathol Oral Radiol Endod. 2004;102(1) :28-34.

2. Kim M, Kim C, Han S, Lee JA. A comparison of three treatment methods for fractures of the mandibular angle. Int J Oral Maxillofac Surg; 2016;45(7):878-83.

3. Morris C, Bebeau NP, Brockhoff H, Tandon R, Tiwana P. Mandibular Fractures: An Analysis of the Epidemiology and Patterns of injury in 4143 Fractures. J Oral Maxillofac Surg. 2015; 73(5):95.

4. Hasegawa $T$, Sadakane $H$, Kobayashi $M$, Tachibana A, Oko T, Ishida $Y$ et al. A multicentre retrospective study of mandibular fractures: do occlusal support and the mandibular third molar affect mandibular angle and condylar fractures?. Int $\mathrm{J}$ Oral Maxillofac Surg. 2016; 45(9):1095-1099.

5. Saito DM, Murr AH. Internal fixation of mandibular angle fractures with the Champy technique. Oper Tech Otolayngol Head Neck Surg. 2008;19(2):123-27.

6. Sugar AW, Gibbons AJ, Patton DW, Silvester KC, Hodder SC, Gray M et al. A randomised controlled trial comparing fixation of mandibular angle fractures with a single miniplate placed either transbuccally and intra-orally, or intraorally alone. Int J Oral Maxillofac Surg. 2009; 38(3):241-45

7. Ellis $E$ 3rd. Treatment methods for fractures of the mandibular angle. Int $\mathrm{J}$ Oral Maxillofac Surg. 1999;28(4):243-52.

8. Al-Moraissi EA, Ellis E. What method for management of unilateral mandibular angle fractures has the lowest rate of postoperative complications? A systematic review and metaanalysis. J Oral Maxillofac Surg. 2014;72(11): 2197-211.

9. Paza AO, Abuabara A, Passeri LA. Analysis of 115 Mandibular Angle. J Oral Maxillofac Surg. 2008; 66(1):73-6.

10. Braasch DC, Abubaker AO. Management of mandibular angle fracture. Oral Maxillofac Surg Clin North Am. 2013;25(4):591-600.

11. Michelet FX, Deymes J, Dessus B. Osteosynthesis with miniaturized screwed plates in maxillo-facial surgery. J Maxillofac Surg. 1973;1(2):79-84. 
12. Champy M, Loddé JP, Schmitt R, Jaeger JH, Muster D. Mandibular osteosynthesis by miniature screwed plates via a buccal approach. J Maxillofac Surg. 1978;6(1):14-21

13. Laverick S, Siddappa $P$, Wong $H$, Patel $P$, Jones DC. Intraoral external oblique ridge compared with transbuccal lateral cortical plate fixation for the treatment of fractures of the mandibular angle: prospective randomised trial. Br J Oral Maxillofac Surg. 2012;50(4):344-9.

14. Seemann R, Schicho K, Wutzl A, Koinig G, Poeschl WP, Krennmair G, et al. Complication rates in the operative treatment of mandibular angle fractures: a 10-year retrospective. J Oral Maxillofac Surg. 2010;68(3):647-50.

15. Ma'aita J, Alwrikat A. Is the mandibular third molar a risk factor for mandibular angle fracture? Oral Surg Oral Med Oral Pathol Oral Radiol Endod. 2000;89(2):143-6.

16. Choi BH, Kim KN, Kang HS. Clinical and in vitro evaluation of mandibular angle fracture fixation with the two-miniplate system. Oral Surg Oral Med Oral Pathol Oral Radiol Endod. 1995; 79(6):692-95

17. Ellis E, Walker L. Treatment of mandibular angle fractures using two noncompression miniplates. J Oral Maxillofac Surg. 1994; 52(10):1032-36.

18. Kuriakose MA, Fardy M, Sirikumara M, Patton DW, Sugar AW. A comparative review of 266 mandibular fractures with internal fixation using rigid (AO/ASIF) plates or mini-plates. $\mathrm{Br} \mathrm{J}$ Oral Maxillofac Surg. 1996;34(4):315-21.

19. Valentino J, Marentette LJ. Supplemental maxillomandibular fixation with miniplate osteosynthesis. J Otolaryngol Head Neck Surgy. 1995;112(2):215-20.

\section{CONFLITO DE INTERESSES}

Os autores declaram não haver conflitos de interesse

\section{AUTOR PARA CORRESPONDÊNCIA}

\section{Éwerton Daniel Rocha Rodrigues}

Faculdade de Odontologia - Universidade de Pernambuco Hospital Universitário Oswaldo Cruz

Departamento de Cirurgia e Traumatologia

Bucomaxilofacial

Rua Arnobio Marques 310 - Santo Amaro

50100-130 Recife - PE, Brasil

E-mail: ewertondaniel27@hotmail.com 